Tordecilla J. y cols.

Rev. Chil. Pediatr. 65 (5); 260-263, 1994

\title{
Alta precoz de niños con cáncer y neutropenia febril
}

\author{
Juan Tordecilla C. ${ }^{\text {; }}$ Myriam Campbell B. '; \\ Pilar Joannon S.' ; Natalie Rodríguez R.'
}

\section{Neutropenia and ferer}

Frorr. april 1982 io july 1993,84 episodes oi lever and neutropen were recorded in 50 oncolog:c patients 139 with acute lymphoblosric leukemia, three with acute myeloid leukenia and e ght with diferen' sclid tumors! which were admitted $10 \mathrm{c}$ children's university associaled public hosplal al Scrntiago Chile. Patients were discharged early after admission if they became non febrile, appeared well. had negarive blood cullures and hed normal chest $x \cdot$ ray in spite of absolute neutrophile courits still under 500 per $\mathrm{mm}^{3}$ I -30 episodes of fever and neutropenia $135,7 \%$ parenteral antibiotic therapy was discontinued and patients were discharged a mean 51 days of hospltaizaton, even while 19 parients still had neutropenia in 17 episodes $\{20,2 \%$, introvenous ant biotic treatmenl was d scontinued and patients were dischorged under oral artibiofic therapy due to minor basteriol infiections aher mecn 01 doys of odmission, while len of these palients were still neulropenic. In 37 es sodes (44.1\%) longer hospitolization ime was

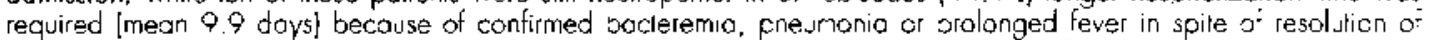
neutropenia. No neutropenic patient at disctarge required readm ssio- to -aspiat within the next seven days and none of them died. Some low risk patients with cancer and febri e neutropenia can be discharged early from hospital in spite of neutropenio.

Kay words: neoplasm, neuropenia, lever.

La hospitalización de niños con cáncer durante los episodjos de neutropenia febril es frecuente y perentoria si el recuento absoluto de neutrofilos en menor de 500 por $\mathrm{mm}^{3}$. El riesgo de infecciones severas está estrechamente rclacionado con la intensidad de la neutropenia, por lo que hay acuerdo sobre el uso precoz de antibióticos de amplio espectro cuando estos pacientes tienen fiebre. Lo corriente es mantener el tratamiento hasta que el niño esté afebril y el recuento de neutrófilos sea mayor de 500 por $\mathrm{mm}^{3}$. Algunos sugieren que el tratamiento antibiótico puede ser suspendido precozmente en ciertos pacientes, todavía neutropénicos, que clínicamente están bien, sin fiebre por 48 horas, con hemocultivos negativos y signos de recuperación hematológica, a los que califican en bajo riesgo ${ }^{1-8}$. Se describe una experiencia en casos de neutropenia febril, a los que se aplić una conducta terapéutica diferenciada con el objeto de identificar los pacientes que podrían beneficiarse con alta precoz.

1. Unidad de Hematologia y Oncología, Hospital Roberto del Río.

\section{Material y Método}

Entre el I de abril de 1992 y el 3l de julio de 1993 ingresaron a la unidad de hematología y oncología del Hospital Roberto del Rio del Servicio de Salud Metropolitano Nonte, Santiago, 50 niños (de 10 meses a 15 años de edad) con neutropenia febril, de los cuales 39 padecian de leucemia línfoblástica aguda (LLA), tres de leucemia mieloide aguda (LMA) y ocho de tumores sólidos. Los nit̂os con leucemia estaban recibiendo en forma profilactica sulfatrimetoprim y nistatina oral. Todos fueron hospitalizados cuando presentaron un episodio de neutropenia (recuento absoluto de neutrófilos menor de 500 por $\mathrm{nm}^{3}$ ) y fiebre ( $T^{\circ}$ axilar $38^{\circ}$ o más en dos oportunidades separadas por 4 horas o un registro de $39^{\circ}$ ), realizandoles examen físico completo: radiograffa de tórax; cultivos de sangre. orina, deposición, faringe o de cualquier lesión especifica que hubiese y hemograma. Este último se controló cada $\mathbf{4 8}$ boras y posteriormente de acuerdo a la evolución clínica. Se isició tratamiento triasociado con cloxacilina $200 \mathrm{mg}$ • $\mathrm{kg}$ - día, amikacina $15 \mathrm{mg} \cdot \mathrm{kg}$ - Jía y cefoperazona 150 $\mathrm{mg} \cdot \mathrm{kg}$ - día. Se consideró buena respuesta clínica a la desaparición de la fiebre dentro de las primeras 72 horas de iniciado el tratamiento ${ }^{7}$. En caso de persistencia de la fiebre se tomaron nuevos cultivos y se adecuaron o cambiaron los antibióticos, tomando en cuenta los cultivos iniciales y las manifestaciones clínicas. Si al séptimo día de evolución el niño seguía con fiebre y neutropenia y no se había identificado la localización de la infección, se agregó anfolericina $B$ al tratamiento. 
La conducta tempéutrca diferenciada consistió en suspender los antrbióticus y dar de alta a los pacientes sin foco identificado de infección. que mostraron huena respaesta clinica, cumplían dos días sin fiebre, sus cultivos resultaban atgatjvos. la radiografía de torax era normal y mostraban signos de recuperación hematológica, aunque persistiera la veutropenia. En los nitos con foco infeccioso identificado, cuyos demás criterios eran similares a los anteriores, incluyeodo signos de recuperación hematológica. aunque persistiera la neutropenia, se suspendian los antibióticos intravenosos y se daba de alta con antibióticos por vía oral, de acuerdo con el tipo y ubicación de la infección que sufritsen. Los pacientes con neumonia. bacteremia o infeccion de una vía yenosa central. se trataron durante diez días ':on antibióticos intravenosos, o por siete días si se registraba recuperación de los neutrofilos y buena respuesta clínica. Los pacientes fueron clasificudos en los diferentes grupos de diagnóstico de acuerdar a definiciones hechas en nuestra expcriencia previay.

\section{Resultados}

Durante los 16 meses de estudio se registraron 84 episodios de neutropenia febril en los 50 pacientes incluidos en el estudio (1,6 ingresos por paciente, márgencs I a 5). La mayotia de los episodios ocurrió en pacientes que estaban en las etapas de diagnóstico (13/64), inducción (25/64) o recaída (23/64) de leucemias linfoides agudas (en cuya fase de mantención sucedieron sólo 3/64); en etapa de inúucción de leucemias mieloides agudas (4/4) y después de la quimioterapia de tumores sólidos (16/16)

Mediante examen físico y estudios de laboratorio fue posible identificar la fuente probable de infección en $51(60,7 \%)$ episodios de leucopenia y fiebre: bacteremia sin localizacion en 19 $(22,9 \%)$, infecciones respiratorias bajas en 15 $(17,9 \%)$, diarrea aguda en $6(9.4 \%)$, infección urinaria en $3(3,6 \%)$. dos episodios de cada uno de tiflitis, celulitis y otitis y uno cada uno de sinusitis y amigdalitis agudas. En los casos de bacteremias se aislaron Staphylococcus coagulasa negativos $(\mathrm{n}=7)$. Staphylococcus aureus $(\mathrm{n}=5)$ Streptococcus grupo D $(\mathrm{n}=1)$, Klebsiella pneumonia, Escherichia coli y Candida atbicans ( 2 de cada una).

La suspensión de los antibióticos con alta de] paciente se aplicó en 30 episodios (35,7\%), 29 de ellos correspondieron a fiebre de origen no precisado y uno a síndrome de diarrea aguda, en 5,1 días -promedio- de estada hospitalaria y con 19 casos que tenían neutropenia. La suspensión del tratamiento antibiótico endovenoso y alta del paciente con antibióticos por vía oral se aplicó en $17 \mathrm{de}$ los 84 episodios $(20,2 \%)$, corespondiendo a bronquitis, diarrea aguda, infección urinaria, otitis aguda, celulitis, sinusitis, amigdalitis y un episodio de fiebre de origen no determinado, con estada hospitalaria promedio de 6,1 días y 10 casos que aún tenían recuentos de neuirbfilos inferiores a 500 por $\mathrm{mm}^{3}$. Se consideró necesario mantener el tratamiento con antibióticos intravenosos por 70 más días en 37 episodios $(44,1 \%)$ que correspondían a bacteremias, neumopatías agudas, tiflitis, diarrea prolongada y uno de fiebre de origen no precisado; con un promedio de 9,9 días de hospitalización y sin neutropenia al alta. No se observaron reingresos de los pacientes en los 7 días siguientes al alta ni fallecimientos.

\section{Comentario}

En los niños con cáncer y neutropenia febril, además de hospitalización y tratamiento con antibióticos, son importantes el recuento absoluto de neutrófilos. la curva térmica y el resultado de los cultivos, pero también los indices de recuperación de la médula ósea y el estado evolutivo de la enfermedad basal, pues sobre estas bases los pacientes puede ser calificados de alto o bajo riesgo, lo que posibilita la aplicación de estrategias diferentes en lo referente a duración del tratamiento con antibióticos ${ }^{8}$.

Los resultados obtenidos en esta experiencia muestran que en más de la mitad de los episodios es posible dar de alta precozmente a los pacientes, aun cuando persiste la neutropenia en algunos de ellos, sin efectos deletéreos detectables, lo que confirma los hallazgos de otros investigadores en pacientes calificados como neutropenia febril de bajo riesgo ${ }^{10.1}$.

La práctica convencional y otros estudios publicados ponen énfasis en que la recuperación de la neutropenia debe ocurrir antes de la suspensión del tratamiento antibiótico por la posibilidad de reinfección de estos pacientes ${ }^{7}, 12,13$. En los casos de alto riesgo es recomendable la hospitalizacion hasta que el recuento absoluto de neutrófilos sea mayor de 500 por $\mathrm{mm}^{3}$ y son los casos de bacteremias, neumontas e infecciones graves y en los que hay pleno acuerdo de tratarlos por períodos del orden de diez o aun más días. 
El alta precoz en los pacientes con leucopenia febril -en especial los de bajo riesgo- tiene ventajas, como menores probabilidades de sobreinfecciones por hongos, agentes resistentes a los antibióticos en uso e infecciones nosocomiales, así como la reducción de los costes de hospitalización. En efecto, para los pacientes incluidos en este análisis, el tiempo de hospitalización disminuyó en total 110 días, lo que representa $30 \%$ de menos de coste ${ }^{14}$.

La ausencia de reingresos hasta siete días después del alta, en los pacientes egresados con neutropenia, y de fallecimientos en el grupo total, confirma que ciertos pacientes con neutropenia febril -cuidadosamente seleccionadospueden ser dados de alta precozmente en forma segura y sugiere que el esquema terapéutico empleado es efectivo.

De esta experiencia se desprende, además, que en niños con cáncer y neutropenia febril es cada vez más frecuente identificar la causa de la fiebre; en sus hemocultivos predominan las bacterias gram positivas; se obtienen buenas respuestas si se emplean esquemas con antibióticos de amplio espectro, basados en las experiencias previas y en la realidad local sobre los agentes causales que se aislan y su sensibilidad y, finalmente, es posible calificar a los afectados como alto o bajo riesgo, de acuerdo a las condiciones clínicas y de laboratorio, para decidir la duración del tratamiento con antibióticos, seleccionando de manera segura a los que pueden beneficiarse de la suspensión de los antibióticos y alta hospitalaria precoz.

\section{Resumen}

Se describe una experiencia en 84 episodios de neutropenia febril, entre abril de 1992 y julio de 1993, en que se aplicó una conducta terapéutica diferenciada, con alta precoz a los niños de buena respuesta clínica, hemocultivos negativos y radiografía de tórax normal, aunque aún exisliese neutropenia. En 30 episodios $(35,7 \%)$ se suspendi6 el tratamiento con antibióticos y se dio el alta al niño, con un promedio de $5, I$ días de estada, cuando todavía había neutropenia en 19 casos. En 17 episodios $(20,2 \%)$ se suspendió el tratamiento endovenoso y se dio de alta al niño con antibioticos por vía oral, por existir un foco definido de infección, con un promedio de
6,1 días de estada y diez casos aún neutropénicos. En 37 episodios $(44,1 \%)$ los pacientes fueron tratados intrahospitalariamente, por un promedio de 9,9 días de estada, porque sufrían de bacteremia, neumonía o fiebre prolongada, hasta la recuperación del número absoluto de neutrófilos. No se registraron reingresos de los pacientes neutropénicos en los siete días siguientes al egreso y no hubo fallecidos en la serie. Algunos pacientes con cáncer y neutropenia febril, que cumplen condiciones para ser catalogados como de bajo riesgo, pueden ser dados de alta en forma precoz aun cuando persista la neutropenia.

(Palabras clave: neoplasmas, neutropenia, fiebre.)

\section{Referencias}

1. Pizzo PA: Evaluation of fever in the patient with cancer. Eur J Clin Oncol 1989; 2/supp] 2:9-16.

2. Pizzo PA, Rubin M, Freifeld A, Walsh TJ: The child with cancer and infection. Enopiric therapy for fever and neutropenia, and preventive strategies. J Pediatr 1991; $119: 679.694$

3. Armsirong $D$ : Empiric therapy for the inmunocompromised host. Rev Infect Dis 1991; 13 (Suppl 9) 763-769.

4. Hathorn JW, and Pizzo PA: Infectious complications in the pediatric cancer patient. IN: Pizzo PA and Poplack DG. Principles and practice of Pediatric Oncology. Philadelphia Lippincott 1989. pp. 837-867.

5. Bodey GP. Buckley $M$. Sathe YS, and Freirich EJ. Quantitative relationship between circulating leucocytes and infection in patients with acute leukemia Ann Intern Med 1966; 64: 328-340.

6. Hughes WT: Empiric antinicrobial therapy in the febrile granulocytopenic patient. Infect Control Hosp Epidemiol 1990; 11: 151-156.

7. Hughes WT, Armstrong D, Bodey GP, et al.: Guide. lines for the use of antimicrobial agents in neutropenic patients with unexplained fever, J Infect Dis 1990; 161: $381-396$.

8. Mullen CA and Buchanan GR: Early hospital discharge of children with cancer treated for fever and neutropenia: Identification and maragement of the low risk patient. J Clin Oncol 1990: 8: 1998-2004.

9. Tordecilla J, Campbell $M$, and Joannon P: Neutropenia febril en niños cáncer. Pediatría (Stgo.) 1992; 35: 218

10. Griffin TC and Buchanan GR: Hematologic predictors of bone marrow recovery in neutropenic patients hospitalized for fever: Implications for disconlinuation of antibiotics and early hospital discharge. J Pediatr 1992: 121: 28-33.

11. Kaplan AH, Webwer DI, Davis $L_{\text {r }}$ et al.: Short courses of antibiotics in selected febrile neutropenic patients. Am J Med Sci 1991: 302: 353-357. 
12. Cohen $K$, Stork $L$, Cullen J. et al.; Discontinuation of antibiotics before neutrophil tecovery in patients with febrile neutropenia (FN) and no identifiable source. Proc Am Soc Clin Oncol 1992; 12: 380.

13. Barson WJ. Brady MT: Management of infections in children with cancer. Hematol Oncol Clin North Am 1987; 1: 801-839.

14. Buchanan GR: Approach to treatment of the febrile cancer patient with low-risk neutropenia. Hematol Oncol Clin North Am 1993; 7:919-935

\section{AVISO A LOS AUTORES}

Cor el objeto de dar prioridad a los trabajos de investigación, en vista de las limitaciones de espacio de la Revista Chilena de Pediatría, el Comité Editorial ha acordado restringir la impresión de casos clínicos a un máximo de dos por cada número. 